OPEN ACCESS

Edited by:

Markus Roth

Albert Ludwigs Universität Freiburg,

Germany

Reviewed by:

Robert Szabo,

Hungarian Academy of Sciences

(MTA), Hungary

Sergei Zharkov,

University of Hull, United Kingdom

${ }^{*}$ Correspondence:

Konstanze Zwintz

konstanze.zwintz@uibk.ac.at

Specialty section

This article was submitted to

Stellar and Solar Physics,

a section of the journal

Frontiers in Astronomy and Space

Sciences

Received: 05 April 2019

Accepted: 11 October 2019

Published: 13 December 2019

Citation:

Zwintz K (2019) The Power of Asteroseismology for Early Stellar

Evolution.

Front. Astron. Space Sci. 6:68. doi: 10.3389/fspas.2019.00068

\section{The Power of Asteroseismology for Early Stellar Evolution}

\author{
Konstanze Zwintz* \\ Institut für Astro- und Teilchenphysik, Universität Innsbruck, Innsbruck, Austria
}

Stars are the building blocks of planetary systems, clusters, associations, and galaxies. The evolution of stars is driven by physical processes in their interiors making theory of stellar interior structure and evolution an important ingredient of contemporary astrophysics. Despite its importance, this theory contains major shortcomings starting from the early stages of stellar evolution which significantly impact all subsequent evolutionary phases. Studying the pulsations of young intermediate-mass stars, i.e., conducting pre-main sequence (pre-MS) asteroseismology, has the potential to contribute to a better understanding of the processes acting during the earliest phases of stellar evolution. With ultra-precise observational data obtained from space and from ground in combination with improvements of our theoretical models for pre-MS stars, the field of pre-MS asteroseismology will advance in the future and provide important constraints for the input physics of early stellar evolution.

Keywords: early stellar evolution, pre-main sequence, $\mathrm{p}$ - and g-mode pulsations, $\delta$ Scuti, $\gamma$ Doradus, SPB, solar-like, space telescopes

\section{INTRODUCTION}

The earliest phases in the lives of stars determine their whole future fate until their deaths. Depending on their initial mass and metallicity, stars produce chemical elements during their further evolution, which can be used to trace the history of Galactic evolution. Massive stars are particularly important for the chemical enrichment of our Universe. The angular momentum that stars obtain during their formation may crucially influence their future lives. These examples illustrate that understanding the physical processes occurring during the earliest stages of stellar evolution is essential. But although we have a general concept of how stars are formed and evolve, our current knowledge of these early phases is very limited.

Asteroseismology has been proven to be a successful tool to unravel details of the internal structure for different types of stars in various stages of evolution. In the mid 1990s it became evident through first time series observations of Herbig Ae stars that also stars in their earliest phases of evolution, i.e., before the onset of hydrogen core burning, can become unstable to pulsations. Therefore, asteroseismology can be successfully applied to pre-main sequence (preMS) stars and will allow us to answer some of the yet open questions of early stellar evolution. In this context, two main challenges need to be tackled in the coming years: we need precise enough observational data for pre-MS pulsators (i.e., long and high precision photometric time series and high-resolution spectroscopy) and improved input physics for the theoretical models of stars before the onset of hydrogen-core burning. 


\subsection{Early Stellar Evolution}

Stars are born from the gravitational collapse of molecular clouds. The formation process is hidden by the obscuring gas and dust until the proto-star has reached sufficient temperature, mass and luminosity to be seen. During this time the protostar continues to accrete matter from its birth cloud. Once the young star emerges from its cocoon, it is suggested by stellar evolution theory to be nearly fully convective and chemically homogeneous. As the central temperature and density are not yet high enough to initiate nuclear burning, the early star gains all of its energy from gravitational contraction. It is in the gas and dust debris disks surrounding the early stars, that planetary systemssimilar to our own solar system-are formed. The relatively short time span between the formation of stars from interstellar clouds and the onset of core hydrogen burning is called the pre-main sequence (pre-MS) phase.

The distribution of stars in young associations (e.g., in the Orion nebula or NGC 2264; Stahler and Palla, 2005) gives the impression that there is an upper boundary in the Hertzsprung-Russell (HR-) diagram, i.e., that pre-MS stars by their very nature are restricted to relatively modest luminosities and that the upper parts are unpopulated. This boundary is named the birthline. Hence, pre-MS stars are the optically visible descendents of embedded protostars. At the birthline, pre-MS stars have protostellar radii that are larger than the main sequence values but never by more than a factor of ten (Stahler and Palla, 2005).

The theoretical representation of the birthline is constructed by calculating sequences of stellar models that represent the youngest pre-MS stars of their respective masses and connecting them. Obviously, the location of the theoretically computed birthline strongly depends on the underlying protostar and pre-MS models, and, therefore, also on the input physics of early stellar evolution that are in turn dependent on different assumptions (e.g., mass accretion rates, angular momentum transfer or turbulence). Following Stahler and Palla (2005) and using theoretical models computed with the MESA (Paxton et al., 2011) or YREC (Demarque et al., 2008) codes, the birthline and the ZAMS intersect at $\sim 6 M_{\odot}$. Other stellar evolution codes such as the CESAM code (Morel and Lebreton, 2008) suggest that the intersection point lies at $\sim 15 M_{\odot}$. This large discrepancy demonstrates our shortcomings in the description of early stellar evolution. If stars more massive than $\sim 6 M_{\odot}$ would indeed have an optically visible pre-MS phase, it would change the lifetimes and ages of more massive stars and, hence, have a strong impact on our concept of how massive stars evolve during their later stages.

The more massive the star, the faster it completes its evolution process. Therefore, the most massive protostars evolve so fast that they do not have an observable pre-MS phase but remain deeply embedded in the remnants of their birth clouds from which they still accrete material. Once they become optically visible, massive stars already burn hydrogen in their cores and, hence, are main sequence objects.

From the birthline on, the pre-MS star's interior structure undergoes several changes as the temperature rises and the opacities decrease (e.g., Stahler, 1983). As the star heats up and the opacities drop, a radiative core develops (Hayashi, 1961). Eventually, the star's central regions become hot enough for nuclear burning to occur, called the main-sequence phase. Models predict that for intermediate mass stars just before the main sequence phase, there is a brief period of nuclear burning where carbon is burnt by the CNO cycle (a chain of hydrogen burning reactions catalyzed by the presence of $\mathrm{C}, \mathrm{N}$, and $\mathrm{O}$ ) until $\mathrm{C}$ and $\mathrm{N}$ reach nuclear burning equilibrium. During this time the star's core briefly becomes convective and the star's luminosity increases. Shortly thereafter, nuclear burning stops and the star resumes its contraction onto the zero-age main sequence (ZAMS), where upon nuclear burning of hydrogen reignites and becomes the dominant source of energy throughout most of the rest of the star's life. These first non-equilibrium nuclear burning processes are reflected in the bending of the pre-MS tracks close to the ZAMS.

The ignition of nuclear fusion is the most important phase in early stellar evolution as it changes the stars' internal structures and marks a key point of their evolution.

\subsection{Pre-main Sequence Stars}

Depending on their masses, pre-MS objects can either belong to the group of $\mathrm{T}$ Tauri stars or show Herbig Ae/Be type characteristics. Members of both groups show photometric and spectroscopic variability on time scales from minutes to years, indicating that stellar activity begins in the earliest phases of stellar evolution, prior to the arrival on the main sequence. Investigating pre-MS members of young open clusters (i.e., younger than 10 million years) improves the a priori identification of stellar youth because all members of young clusters have the same formation and early evolution history, and therefore cannot be confused with more evolved foreground or background objects.

\subsection{T Tauri Stars}

Once a low-mass star $\left(0.2 M_{\odot} \leq \mathrm{M} \leq 1.5 M_{\odot}\right)$ becomes optically visible and contracts along its Hayashi track, it presumably becomes a T Tauri star. T Tauri stars were discovered by Joy $(1942,1945,1949)$ in the Taurus-Auriga dark cloud and named after their brightest member. They are primarily of spectral types from late $\mathrm{F}$ to $\mathrm{M}$ and possess apparently normal photospheres with overlying continuum and line-emission characteristics of a hotter (say 7,000-10,000 K) envelope. Several studies (e.g., Joy, 1945, 1949; Herbig, 1962) established beyond doubt that these stars are in their pre-MS phase of evolution.

Weak-lined T Tauri stars (WTTSs) do not show evidence for disk accretion. Their sinusoidal light variability is attributed to cool magnetic spots on the stellar surface (e.g., Stassun and Wood, 1999; Cody et al., 2014). Classical T Tauri stars (CTTSs) are surrounded by often dense circumstellar disks from which matter is accreted. Consequently, their time domain behavior is much more complex and can be categorized as being stochastic (e.g., Rucinski et al., 2008), intermittently fading (e.g., Alencar et al., 2010), or semi-periodic (e.g., Alencar et al., 2010). The physical mechanisms causing aperiodic or partially periodic variability include obscuration by circumstellar matter (e.g., Alencar et al., 2010), instabilities in the accration shock at the 
stellar surface (Koldoba et al., 2008), unsteady accretion and hot spot evolution (e.g., van Boekel et al., 2010), and instabilities in the accretion disk (e.g., Bouvier et al., 2007). But also magnetic field strength and shape (e.g., Cody et al., 2014; Stauffer et al., 2016), disk structure (e.g., Wolk et al., 2013), stellar mass (Herbst and Shevchenko, 1999), and rotation rate (Grankin et al., 2007) also appear to influence the variability of CTTSs. A detailed overview is given in Cody et al. (2014).

Alencar et al. (2010) identified three types of CTTS light curves using data obtained by the CoRoT satellite (Auvergne et al., 2009) during the Short Run SRa01 on the young open cluster NGC 2264: regular spot-like variability, semi-regular AA Tau like stars and irregular light variations. Their study was continued with the second observing run of CoRoT on NGC 2264 during the CSI2264 project ["Coordinated Synoptic Investigation of NGC 2264"; Cody et al. (2014)] in 2011/12. Recently, Stauffer et al. (2016) used these data for a new morphological classification of the CTTSs into six groups: (i) periodic extinction variability, (ii) aperiodic extinction variability, (iii) unstable accretion or accretion bursts, (iv) cold spots, (v) hot spots and (vi) stochastic variability.

The regular and irregular light variability typical for $\mathrm{T}$ Tauri stars can be measured spectroscopically and photometrically and is complicating the search for and analysis of the pulsational signal in these stars. In the effective temperature range covered by $\mathrm{T}$ Tauri objects, pulsational periods are expected to be as long as a few days in case of $\gamma$ Doradus type pulsation. Consequently, variability caused by the disk and caused by pulsations lies in the same domain and is hard to disentangle. But also stochastic solar like oscillations are predicted in this region of the HR diagram which have significantly shorter periods on the order of a few to few tens of minutes, but have amplitudes on the parts-per-million level which are observationally hard to detect in light curves that are dominated by aperiodic variability.

\subsection{Herbig Ae/Be Stars}

In the case of intermediate-mass stars $\left(1.5 M_{\odot} \leq \mathrm{M} \leq 10 M_{\odot}\right)$ the imprint of the previous accretion history persists much longer and their evolution is much closer tied to the protostellar conditions than for low-mass stars. The star's surface luminosity increases sharply early during contraction. Also, the star inherits a thick, subsurface mantle of deuterium, which must ignite in a shell and fuse to helium during the subsequent approach to the ZAMS. The lower mass limit of $1.5 \mathrm{M}_{\odot}$ corresponds to the mass above which stars are radiatively stable when they begin their quasistatic contraction. The upper limit $\left(10 M_{\odot}\right)$ corresponds to the mass above which stars start burning hydrogen before they emerge from their contracting envelope, i.e., it occurs where the stellar birthline (Stahler, 1983) intersects the ZAMS.

Intermediate-mass pre-MS stars are attributed to the group of Herbig Ae/Be stars (Herbig, 1962) which have spectral types A or $\mathrm{B}$ and show emission lines. They possess an IR excess due to hot or cool circumstellar dust or both, and have luminosity classes III-V (Waters and Waelkens, 1998). The difference between Herbig Ae/Be and "normal" main sequence stars is the presence of emission lines and the complex variability of the emission and absorption features. Using the shapes of the $\mathrm{H} \alpha$ emission lines,
Reipurth et al. (1996) classified the stars into four groups: Type I objects show symmetric profiles without absorption features; the emission lines of Type II stars are double-peaked where the secondary peak has more than half the strength of the primary; in the group of Type III objects the profiles are double-peaked, but the secondary peak has less than half the strength of the primary peak; Type IV has the classical P Cygni profile (see e.g., Vieira et al., 2003).

The light variability observed in Herbig Ae/Be stars ranges from days to weeks (e.g., Oudmaijer et al., 2001) in period and reaches up to a magnitude in strength. Recent studies show that within the mass range of Herbig Ae/Be stars the accretion mechanism changes from being magnetically controlled as in T Tauri stars (Bouvier et al., 2007) to a currently unknown mechanism acting in the more massive stars. The light variability observed in Herbig Ae/Be stars originates from variable extinction, from rotating circumstellar disks, or from rotation of cold photospheric spots.

In the effective temperature range of Herbig Ae/Be stars, pmode $\delta$ Scuti variability with periods ranging from $18 \mathrm{~min}$ to $5 \mathrm{~h}$ can be found. As the p-mode periods are on a much shorter time scale than any other variability seen in Herbig Ae/Be stars, they can be identified somewhat easier as is illustrated in section 4.2.

\section{FIRST STEPS IN ASTEROSEISMOLOGY OF PRE-MS STARS}

The first reference to the presence of pulsations in a preMS star dates back to a light variability study of the young open cluster NGC 2264, where Breger (1972) reported the two stars V 588 Mon (HD 261331, NGC 22642) and V 589 Mon (HD 261446, NGC 226420) to show $\delta$ Scuti -like oscillations. The position of the two stars in the HR-diagram agreed with that of post-main sequence $\delta$ Scuti stars, but given the extremely young age of NGC 2264 of 1-3 Myr, Michel Breger concluded in a visionary statement in his publication that "... if they are members, they would be the first known pre-main sequence pulsators" (Breger, 1972). Consequently, the membership of the two stars to the cluster was investigated and discussed in subsequent years.

Only 20 years later, the next pre-MS pulsator was discovered: HR 5999 (HD 144668, V 856 Sco) is a Herbig A7 III-IVe star that was intensively studied in connection to its HerbigAe-nature (e.g., Praderie et al., 1991) until then. None of the studies was aiming to detect oscillations because at that time it was not clear if stars in their earliest phases of evolution could indeed show similar pulsational properties as their more evolved, hydrogen core burning counterparts. Kurtz and Marang (1995) detected clear $\delta$ Scuti -type variability with a period of about $4.99 \mathrm{~h}$ with an amplitude of only 13 mmag in the presence of 0.35 mag nonperiodic light variations in HR 5999. The authors also conducted the first detailed asteroseismic investigation of the oscillations in a pre-MS star. Hence, this marks the real start of the field of pre-MS asteroseismology.

In the late 1990ies a few more objects were identified as being potentially pre-MS pulsators, i.e., HD 104237 (Kurtz and 
Müller, 1999), V 351 Ori and HD 35929 (Marconi et al., 2000), NGC 6823 HP 57 and NGC 6823 BL 50 (Pigulski et al., 2000). At the same time, the first investigation of the theoretical instability strip for pre-MS $\delta$ Scuti stars was published (Marconi and Palla, 1998) illustrating that the main difference between a pre- and a post-MS star of same mass and location in the HR-diagram lies in its interior structure. In these early stages of the research area, pre-MS stars were believed to be purely radial pulsators showing only few $\delta$ Scuti -type pulsation modes (Marconi and Palla, 1998).

In subsequent years, the number of confirmed detections of $\delta$ Scuti-type pulsations in pre-MS stars increased first using ground-based observations (e.g., Zwintz et al., 2005; Ripepi et al., 2006) followed by the first space data obtained from the MOST satellite (Walker et al., 2003). It soon became evident that preMS pulsators can show both, radial and non-radial pulsation modes (Ruoppo et al., 2007; Zwintz et al., 2007) and can be as multi-periodic as their more evolved counterparts showing more than a handful and up to more than 100 individual pulsation frequencies (e.g., Kallinger et al., 2008; Zwintz et al., 2009). A detailed investigation of the pulsation frequency spectra of preand post-MS $\delta$ Scuti -type stars clearly illustrated that the excited frequencies differ between the two evolutionary stages, hence making it possible to constrain the evolutionary stage of a given star by its pulsation properties only, if a sufficient number of oscillations modes was detected observationally (Suran et al., 2001). Also, the first theoretical pre-MS pulsation models were successfully applied for the determinination of the pulsational characteristics of the observed frequencies (e.g., Guenther et al., 2007, 2009). This also allowed for the first time to constrain the evolutionary stage of a star only by using its pulsational properties (Guenther et al., 2007).

\section{CURRENT STATE-OF-THE ART IN PRE-MAIN SEQUENCE ASTEROSEISMOLOGY}

As of January 2019, $\sim 75$ pre-MS stars were discovered to be $p$ and $g$-mode pulsators of three different types.

\subsection{Pre-main Sequence $\delta$ Scuti Stars}

Similar to their hydrogen core burning counterparts, pre-MS $\delta$ Scuti pulsators are intermediate mass stars of spectral types A-F with pulsation periods between about $18 \mathrm{~min}$ and $7 \mathrm{~h}$ and pulsation amplitudes at the millimagnitude level. $\delta$ Scuti stars in all evolutionary stages pulsate with $p$-modes that are driven by the $\kappa$-mechanism in the $\mathrm{H}, \mathrm{He} \mathrm{I}$, and He II ionization zones.

Recently, a relation between the oscillatory properties of $\delta$ Scuti-type pre-MS stars and the relative stage in their pre-MS evolution was revealed (Zwintz et al., 2014). The least evolved stars that are located close to the birthline and hence have the largest radii, show the slowest pulsations. The further the preMS stars have progressed in their evolution toward the ZAMS, and hence the more compact they have become, the faster they oscillate. A relation like this has so far only been shown for the stochastically excited solar-like oscillations in red giants (e.g., Bedding et al., 2011) - a completely different type of pulsations in a different evolutionary stage. Being able to link the relative ages of heat-driven $\delta$ Scuti -type pre-MS stars to their pulsational properties is the first step in using asteroseismic methods to unravel open questions in early stellar evolution such as the preMS lifetimes, the speed of stellar evolution or the chemical and rotational evolution of intermediate-mass pre-MS stars.

\subsection{Pre-main Sequence Slowly Pulsating B (SPB) Stars}

SPB stars have spectral types from B3 to B9, pulsate with $g$-modes excited by the $\kappa$-mechanism and show periods between 0.5 and 3 days (Aerts et al., 2010). SPB stars with 2-7 $M_{\odot}$ are more massive than the $\delta$ Scuti stars, and, hence have a significantly shorter pre-MS phase lasting only a few million years.

Pre-MS SPB stars are already in the crucial transition phase from gravitational contraction to hydrogen-core burning where the star undergoes significant structural changes before arrival on the ZAMS. These changes should be measurable by detecting regularities in period and their deviations from an equal spacing similar as it has been recently successful for main sequence SPB stars (e.g., Pápics et al., 2015). As pre-MS SPB stars have a much shorter optically visible pre-MS evolutionary phase, they are statistically less frequent, and hence harder to find.

Gruber et al. (2012) reported the first possible detection of two pre-MS SPB stars: GSC 00154-00785 and GSC 00154-01871 are two members of the young open cluster NGC 2244. A detailed analysis of B type members in the young cluster NGC 2264, revealed SPB type pulsations in four stars (HD 47469, HD 48012, HD 261810, and HD 261878), but identified them to be already on the ZAMS with ages of only $\sim 1$ to 2 million years (Zwintz et al., 2017). This illustrates the challenges to be met when searching for SPB type pulsations in pre-MS stars: the time stars of four to five solar masses need to start burning hydrogen in their cores is quite short lasting only a few ten-thousand years. This makes it hard to detect true pre-MS SPB stars.

\subsection{Pre-main Sequence $\gamma$ Doradus Stars}

Pre- and (post-)MS $\gamma$ Doradus pulsators have periods similar to the SPB stars, i.e., from 0.3 to 3 days, and also pulsate with $g$ modes (e.g., Kaye et al., 1999). Therefore, a reliable value for the effective temperature is crucial to distinguish between SPB and $\gamma$ Doradus pulsation.

The existence of pre-MS $\gamma$ Doradus type stars that are cooler than the $\delta$ Scuti type stars with early $\mathrm{F}$ spectral types and show pulsations driven by convective blocking (Guzik et al., 2000) was first predicted theoretically by Bouabid et al. (2011). These theoretical calculations were conducted without observational evidence. Bouabid et al. (2011) and showed that the exact location of the borders of the instability region strongly depends on the treatment of convection for which mixing length theory (BöhmVitense, 1958) was used. The mixing length parameter, $\alpha$, is usually determined by solar models and can have values between $\sim 1.2$ and 2.2. The smaller the value for $\alpha$, the more the borders of the pre-MS $\gamma$ Doradus instability strip will shift toward cooler temperatures, hence illustrating the importance of the treatment of convection for stars in this temperature range. Constraints will 
come from observations of a significant number of $\gamma$ Doradus type objects in their pre-MS evolutionary stage.

The few currently known g-mode pre-MS $\gamma$ Doradus type objects (Zwintz et al., 2013) show pulsation periods between roughly 0.2 and 3 days and are of particular interest in context with the formation of planets around young stars due to their cooler temperatures (Udry and Santos, 2007).

\subsection{Theoretical Framework}

The theoretical framework of pre-MS asteroseismology is currently lagging behind the observational findings and so far only the first steps have been taken.

For $\delta$ Scuti type stars, Suran et al. (2001) illustrated for the first time on a theoretical basis how different pulsation frequency patterns are expected between pre-MS and main sequence objects of same mass. Guenther et al. (2007) provided the first evidence that the evolutionary stage of a given $\delta$ Scuti type star can indeed be identified through the analysis of its pulsation modes: for the star NGC $6530 \mathrm{ZW} 278$ the p-modes alone allowed to differentiate between the pre- and the post-MS stages and clearly illustrated its pre-MS nature.

Other studies focused on fitting theoretical nonradial p-mode oscillation spectra computed from dense grids of pre-MS models to the observed oscillation spectra of pre-MS stars. Some of these fits could nicely yield a mode-identification and asteroseismic interpretation while others fail to yield a good match between theoretical model and observational frequencies (e.g., some stars in NGC 2264 can be modeled well, others cannot; Guenther et al., 2009). The reasons why for some stars the models do not reach satisfactory results are manifold and include the lack of a proper description of accretion or convection in the theoretical models or incorrect observational parameters that are used to constrain the stars' positions in the HR diagram (e.g., luminosity).

\section{CHALLENGES FOR PRE-MS ASTEROSEISMOLOGY}

\section{The investigation of pulsating pre-MS stars contains several challenges:}

\subsection{Ambiguity in the Evolutionary Stage}

Intermediate mass pre-MS stars share the same location in the HR-diagram as their evolved counterparts in the (post-)MS phase that already burn hydrogen in their cores in full equilibrium. Pre- and post-MS stars of same effective temperature $\left(T_{\text {eff }}\right)$, luminosity $\left(L_{\odot}\right)$ and mass have very similar atmospheric properties and mostly differ in their interior structures (Marconi and Palla, 1998). It is therefore not possible to determine the evolutionary stage of a given star unambiguously only by its position in the HR-diagram.

In general, the evolutionary stage of a star in this region of the HR diagram can only be constrained using some observational features that are important indicators for stellar youth: emission lines in the spectra, infrared or ultraviolet excesses, high X-ray fluxes, and membership either to a very young open cluster (i.e., younger than a few million years) or to a star forming region. But these features can also be misleading, as for example the comparatively old, low-mass asymptotic giant branch (AGB) and post-AGB stars show similar observational properties (e.g., infrared excess and emission lines) and populate the same region in the HR diagram as young stellar objects (e.g., Kamath et al., 2014).

Therefore, the identification of the pre-MS nature of a given star is not straightforward, and an additional criterion providing direct evidence for the early evolutionary stage is needed. As the difference in evolutionary stages of intermediate-mass stars is reflected in quite different interior structures, asteroseismology offers such a criterion.

As illustrated by Suran et al. (2001), different frequencies are excited in a pre- and a post-main sequence star of same mass, effective temperature and luminosity resulting in a clearly different frequency pattern in particular for non-radial modes. If observations provide sufficient pulsation frequencies, they can be matched to the theoretical models and lead to an identification of the evolutionary stage of a given star from its observed frequency pattern (e.g., Guenther et al., 2007).

Hence, with a sufficiently large sample of known pre-MS pulsators of different types as calibrators, we will be able to clearly distinguish a young star from its way more evolved counterpart only from its pulsational properties.

\subsection{Activity of Pre-MS Stars}

Young stars are known to show periodic and aperiodic variability caused by inhomogeneities in a circumstellar shell or disk, or variations caused by magnetic effects (e.g., star spots) in the stellar photosphere. In many cases, these variations have longer timescales than the pulsation, but in some cases both types of variability have similar periods (e.g., for SPB or $\gamma$ Doradus type pre-MS stars). For the study of stellar pulsations in the pre-MS phase it is important to distinguish the variability originating from the circumstellar environment from the light variations caused by pulsations. Figure 1 in Zwintz et al. (2009) shows the light curves of HD 142666 observed by the MOST space telescope in 2006 and 2007 as an example. This young star is surrounded by a dense circumstellar disk whose clumpy structure obscures the star and causes light to change irregularly. In the present data no signs of binarity or of the presence of a planet can be seen. But in the circumstellar disk of HD 142666 gaps have been detected which are possibly carved by a young giant planet of $\sim 0.1$ to 0.3 Jupiter masses (Rubinstein et al., 2018).

Additionally, HD 142666 shows $\delta$ Scuti-like pulsations with amplitudes at the millimagnitude level which are only partly revealed when calculating a classical Fourier Analysis of the original light curve (see Figures 2-5 in Zwintz et al., 2009). As can be seen, the time scale of variations from the circumstellar shell mostly affects the low-frequency region of the amplitude spectrum.

Sporadic, rapid effects due to interaction with the shell do not persist in the frequency spectra. Most of the stars with accretion bursts, for example, do not show a significant periodic signal. In their study of young stellar objects in NGC 2264, Stauffer et al. (2014) report that only four stars have strong periodicities, which lie between 6 and 7 days, corresponding to frequencies 
between 1.938 and $1.653 \mu \mathrm{Hz}$. Moreover, in the presence of both, pulsations and rotation, it is often possible to discriminate the frequencies from rotation and pulsation in an additional way in Fourier space: A rotational frequency will sometimes occur with multiples, while this is not the case for the pulsational frequencies in the linear regime (e.g., Pápics et al., 2012; Thoul et al., 2013).

In all asteroseismic studies, special care is needed to disentangle the regular and irregular variability of pre-MS stars originating from the circumstellar disk or spots on the stellar surface from the pulsational variabilities (e.g., Zwintz et al., 2009). In the case of $\delta$ Scuti type oscillations the pulsation frequencies lie in a range well separated from the frequencies caused by the aperiodic shape of the light curve. However, for pulsators with frequencies in the lower regime (e.g., $\gamma$ Doradus and SPB stars) the pulsational signal gets mixed with the peaks originating from the irregular variability and the disentangling of the contributions of the disk and the pulsations gets even more complex.

\subsection{Observational Material}

Space photometry has revolutionized our understanding of main sequence and post-MS pulsators-the best example is the field of solar like oscillations in main sequence dwarfs and red giants. Only with the long enough time bases for the photometric time series as obtained using the space telescopes CoRoT (Auvergne et al., 2009) and Kepler (Borucki et al., 2010), the probing power of solar like oscillations became available and enhanced the field significantly. In the case of pre-MS pulsations, the first space data were obtained using the MOST satellite (Walker et al., 2003) with time bases of up to a month (e.g., Zwintz et al., 2009), followed by two CoRoT Short Runs on the young cluster NGC 2264 which again had a maximum observing length of about 1 month (e.g., Zwintz et al., 2013). Only with Kepler K2 (Gilliland et al., 2010), the time bases for pre-MS observations started to increase to $\sim 78-80$ days. The NASA mission TESS (Ricker et al., 2015) will at least help to increase the observational material for preMS pulsations: Although many of the young regions of the Milky Way will not be observed during the TESS main mission (e.g., NGC 2264), several of the known pre-MS pulsators and candidates will be included in at least one, but up to four sectors. Hence, for a few known pre-MS pulsators, the time bases can reach $\sim 108$ days - which is about the minimum accuracy needed to search for g-mode period spacings. The immediate hope for pre-MS asteroseismology lies in an extended TESS mission that will hopefully also observe the youngest regions of our Milky Way with sufficiently long photometric time series of ultrahigh precision.

\section{FUTURE PERSPECTIVES FOR PRE-MAIN SEQUENCE ASTEROSEISMOLOGY}

Our current general concept of early stellar evolution is widely adopted, but we are still facing many open questions that are connected to missing input physics for our theoretical models and to a lack of observational constraints. I briefly discuss a few selected topics below:

Definition of the birthline: One of these open issues is, for example, the exact location of the birthline as recent studies using different sets of theoretical models suggest that its intersection with the ZAMS might lie at significantly higher masses (i.e., $\sim 15 \mathrm{M}_{\odot}$ Alecian et al., 2013). In this context, observations of the youngest objects over the whole pre-MS mass range to be conducted in the future, will help to test the underlying proto-star and pre-MS theory and provide crucial input for them.

Angular momentum: We also do not completely understand how the angular momentum of stars evolves from their births until they reach the ZAMS and if young stars rotate as solid bodies or show differential rotation between their cores and their envelopes as it was already found in main sequence stars (e.g., Kurtz et al., 2014). The detection and analysis of g-mode period spacings in pre-MS pulsators will put constraints on the angular momentum transport in young stars and connect to the findings for main sequence and post-MS objects.

Chemical peculiarities: In $~ 10 \%$ of stars on the main sequence, we find chemical peculiarities in their atmospheres, but we do not know when these anomalies form, if they are only surface effects or if they reach into the deep stellar interiors. The investigation of surface chemical composition together with the information derived from asteroseismic analyses is expected to contribute to answer these questions.

Pre-MS ages: Another big topic in stellar astrophysics is the definition of an indicator that allows precise determinations of stellar ages, and, in particular the differentiation between preand post-MS stars of same fundamental parameters. Although age is a fundamental tool to understand different phenomena in stellar astrophysics, it can currently be constrained to not better than $\sim 30-40 \%$. In the pre-MS stages, the errors in age can be even up to $100 \%$ : ages of young clusters are mostly given as, e.g., $5 \pm 5 \mathrm{Myr}$. While the precise definition of the zero point in the definition of stellar age is insignificant for most evolutionary phases from the main sequence toward the final stages of stellar evolution, this is not the case during the pre-MS stages: making a systematic error of-say-one million years comprises only a tiny error of $\sim 0.02 \%$ at the current age of our Sun, but would be more than $100 \%$ for a pre-MS late B type star. Hence, one of the future perspectives of pre-MS asteroseismology is the potential to provide an independent age calibrator for stars before the onset of hydrogen burning: If empirical relations like the one found for pre-MS $\delta$ Scuti type stars will exist for the other pre-MS pulsator types as well, we will be able to use the asteroseismic properties of pre-MS stars to provide more reliable age references for the early evolutionary phases.

Pre-main sequence solar like oscillators: All presently known pre-MS pulsators are more massive than the Sun. But our Sun shows stochastic oscillations that are successfully used to constrain its interior structure (Christensen-Dalsgaard, 2002). This is already an indication that stars could also show solar-like oscillations before arrival on the ZAMS, hence being examples for the early Sun. Indeed, theory predicts the existence of these stochastic pulsations in pre-MS objects with $\sim 1 M_{\odot}$ (e.g., Samadi et al., 2005). The observational proof is still missing as such 
low mass pre-MS objects mostly have a high degree of activity which is reflected in regular and irregular light variability on relatively large scales (i.e., up to few magnitudes, e.g., Alencar et al., 2010). Therefore it is not easy to detect the signs of solar like oscillations that are on the order of a few micromagnitudes in such light curves (Samadi et al., 2005). Discovering a "young Sun" is important to test stellar evolutionary codes and to study early planetary evolution, including Earth, with further implications on our understanding of the habitability of other planets.

So far, we have not been able to discover solar-like oscillations in pre-MS stars mainly because the available data sets did not allow to pick up their signal on the micromagnitude level which is buried by the strong variability caused by the activity phenomena in young stars.

Tidally induced pulsations: Many pulsating stars are found in binary systems-two stellar objects that periodically revolve around their common center of mass. The two stars forming a binary system should have been born under the same initial conditions in the molecular cloud, which particularly implies the same initial chemical composition and age of the individual stellar components. If the two stars reside in a close binary orbit, they exert tidal forces similar as the tides exerted by the Moon on the Earth. The strong tidal forces that are generated during the stars' close periastron passages can influence their pulsation properties in two ways: (i) the stars' oscillations get shifted in frequency due to the tidal perturbations, or (ii) tidally induced gravity mode pulsations get resonantly excited by the stars' dynamic tides. Consequently, tidally induced pulsations should also exist in pre-MS stars, but so far no system has been discovered.

Theoretical models: The current theoretical framework of preMS asteroseismology lacks, for example, theoretical models for g-mode pre-MS oscillations in SPB and $\gamma$ Doradus type stars, a refined calculation of the instability regions for all presently known objects and-based on our advanced knowledge of solarlike oscillations in main sequence and post-main sequence stars-a corresponding refined theoretical analysis of predicted solar-like pulsations in pre-MS stars. Work has been already started to overcome the lack of a solid theoretical framework

\section{REFERENCES}

Aerts, C., Christensen-Dalsgaard, J., and Kurtz, D. W. (2010). Asteroseismology. Dordrecht; Heidelberg; London; New York, NY: Springer Verlag. doi: 10.1007/978-1-4020-5803-5

Alecian, E., Wade, G. A., Catala, C., Grunhut, J. H., Landstreet, J. D., Bagnulo, S., et al. (2013). A high-resolution spectropolarimetric survey of Herbig Ae/Be stars - I. Observations and measurements. Month. Notices RAS 429, 1027-1038. doi: $10.1093 / \mathrm{mnras} / \mathrm{sts} 384$

Alencar, S. H. P., Teixeira, P. S., Guimarães, M. M., McGinnis, P. T., Gameiro, J. F., Bouvier, J., et al. (2010). Accretion dynamics and disk evolution in NGC 2264: a study based on CoRoT photometric observations. Astron. Astrophys. 519:A88. doi: 10.1051/0004-6361/201014184

Auvergne, M., Bodin, P., Boisnard, L., Buey, J.-T., Chaintreuil, S., Epstein, G., et al. (2009). The CoRoT satellite in flight: description and performance. Astron. Astrophys. 506, 411-424. doi: 10.1051/0004-6361/200810860

Bedding, T. R., Mosser, B., Huber, D., Montalbán, J., Beck, P., ChristensenDalsgaard, J., et al. (2011). Gravity modes as a way to distinguish between for pre-MS oscillators, because this lack is currently one of the bottlenecks for the future advances of the field.

\section{FUTURE OUTLOOK}

The field of pre-MS asteroseismology started only about 25 years ago with the first asteroseismic analysis of a pre-MS $\delta$ Scuti star (Kurtz and Marang, 1995). Hence, it is a relatively new research area that will definitely grow in the future by upcoming data from space missions such as TESS in combination with supplementary ground-based observations and by triggering the needed improvements of our theoretical models for the preMS stages.

I was personally involved in the development of this field since my $\mathrm{PhD}$ thesis which aimed at finding pre-MS $\delta$ Scuti type pulsators in young open clusters. Since then I am fascinated by the earliest phases of stellar evolution and am now in the lucky position to share my passion for pre-MS pulsations with my students and team members. Consequently, I would definitely take up this field again if I were a student and was offered this as a topic for my $\mathrm{PhD}$ thesis.

From my personal view, I see the field of pre-MS asteroseismology advancing in the future, and I am certain when we learn more about young stars and their evolution, we will face one or the other surprise as well.

\section{AUTHOR CONTRIBUTIONS}

$\mathrm{KZ}$ wrote the complete manuscript.

\section{ACKNOWLEDGMENTS}

This publication was supported by the Publishing fund of the University of Innsbruck. KZ acknowledges support from the Austrian Science Fund (FWF), the Austrian Academy of Sciences (ÖAW), the Austrian Research Promotion Agency (FFG), and the Research Foundation Flanders (FWO) which she received throughout her career. hydrogen- and helium-burning red giant stars. Nature 471, 608-611. doi: $10.1038 /$ nature09935

Böhm-Vitense, E. (1958). Über die Wasserstoffkonvektionszone in Sternen verschiedener Effektivtemperaturen und Leuchtkräfte. Mit 5 Textabbildungen. Zeitschrift Astrophysik 46:108.

Borucki, W. J., Koch, D., Basri, G., Batalha, N., Brown, T., Caldwell, D., et al. (2010). Kepler planet-detection mission: introduction and first results. Science 327:977. doi: 10.1126/science.1185402

Bouabid, M.-P., Montalbán, J., Miglio, A., Dupret, M.-A., Grigahcène, A., and Noels, A. (2011). Theoretical seismic properties of premain sequence $\gamma$ Doradus pulsators. Astron. Astrophys. 531:A145. doi: 10.1051/0004-6361/201116440

Bouvier, J., Alencar, S. H. P., Boutelier, T., Dougados, C., Balog, Z., Grankin, K., et al. (2007). Magnetospheric accretion-ejection processes in the classical T Tauri star AA Tauri. Astron. Astrophys. 463, 1017-1028. doi: 10.1051/0004-6361:20066021

Breger, M. (1972). Pre-main stars. I. Light variability, shells, and pulsation in NGC 2264. Astrophys. J. 171:539. doi: 10.1086/151308 
Christensen-Dalsgaard, J. (2002). Solar g-mode oscillations. Int. J. Mod. Phys. D 11, 995-1009. doi: 10.1142/S0218271802002517

Cody, A. M., Stauffer, J., Baglin, A., Micela, G., Rebull, L. M., Flaccomio, E., et al. (2014). CSI 2264: simultaneous optical and infrared light curves of young diskbearing stars in NGC 2264 with CoRoT and spitzer - evidence for multiple origins of variability. Astron. J. 147:82. doi: 10.1088/0004-6256/147/4/82

Demarque, P., Guenther, D. B., Li, L. H., Mazumdar, A., and Straka, C. W. (2008). YREC: the Yale rotating stellar evolution code. Nonrotating version, seismology applications. Astrophys. Space Sci. 316, 31-41. doi: 10.1007/s10509-007-9698-y

Gilliland, R. L., Brown, T. M., Christensen-Dalsgaard, J., Kjeldsen, H., Aerts, C., Appourchaux, T., et al. (2010). Kepler asteroseismology program: introduction and first results. Publ. ASP 122:131. doi: 10.1086/650399

Grankin, K. N., Melnikov, S. Y., Bouvier, J., Herbst, W., and Shevchenko, V. S. (2007). Results of the ROTOR-program. I. The long-term photometric variability of classical T Tauri stars. Astron. Astrophys. 461, 183-195. doi: 10.1051/0004-6361:20065489

Gruber, D., Saio, H., Kuschnig, R., Fossati, L., Handler, G., Zwintz, K., et al. (2012). New slowly pulsating B stars in the field of the young open cluster NGC 2244 discovered by the MOST photometric satellite. Month. Notices RAS 420, 291-298. doi: 10.1111/j.1365-2966.2011.20033.x

Guenther, D. B., Kallinger, T., Zwintz, K., Weiss, W. W., Kuschnig, R., Casey, M. P., et al. (2009). Asteroseismic analysis of the pre-main-sequence stars in NGC 2264. Astrophys. J. 704, 1710-1720. doi: 10.1088/0004-637X/704/2/1710

Guenther, D. B., Kallinger, T., Zwintz, K., Weiss, W. W., and Tanner, J. (2007). Seismology of pre-main-sequence stars in NGC 6530. Astrophys. J. 671, 581591. doi: $10.1086 / 522880$

Guzik, J. A., Kaye, A. B., Bradley, P. A., Cox, A. N., and Neuforge, C. (2000). Driving the gravity-mode pulsations in $\gamma$ doradus variables. Astrophys. J. Lett. 542, L57-L60. doi: 10.1086/312908

Hayashi, C. (1961). Stellar evolution in early phases of gravitational contraction. Publ. Astron. Soc. Jpn. 13, 450-452.

Herbig, G. H. (1962). The properties and problems of $\mathrm{T}$ Tauri stars and related objects. Adv. Astron. Astrophys. 1, 47-103. doi: 10.1016/B978-1-4831-9919-1.50006-6

Herbst, W., and Shevchenko, V. S. (1999). A photometric catalog of herbig AE/BE stars and discussion of the nature and cause of the variations of UX orionis stars. Astron. J. 118, 1043-1060. doi: 10.1086/300966

Joy, A. H. (1942). Spectral criteria in the classification of variable stars. Publ. Astron. Soc. Pacific 54:15. doi: 10.1086/125386

Joy, A. H. (1945). T tauri variable stars. Astrophys. J. 102:168. doi: 10.1086/144749

Joy, A. H. (1949). Bright-line stars among the Taurus dark clouds. Astrophys. J. 110:424. doi: $10.1086 / 145217$

Kallinger, T., Zwintz, K., and Weiss, W. (2008). Pulsating pre-MS stars in the young open cluster NGC 2264: V588 Monocerotis and V589 monocerotis. Astron. Astrophys. 488, 279-286. doi: 10.1051/0004-6361:20079207

Kamath, D., Wood, P. R., and Van Winckel, H. (2014). Optically visible post-AGB/RGB stars and young stellar objects in the Small Magellanic Cloud: candidate selection, spectral energy distributions and spectroscopic examination. Month. Notices R. Astron. Soc. 439, 2211-2270. doi: 10.1093/mnras/stt2033

Kaye, A. B., Henry, G. W., Fekel, F. C., and Hall, D. S. (1999). Simultaneous photometry and spectroscopy of the newly discovered $\gamma$ Doradus variable HR $8330=$ HD 207223. Month. Notices R. Astron. Soc. 308, 1081-1086. doi: 10.1046/j.1365-8711.1999.02768.x

Koldoba, A. V., Ustyugova, G. V., Romanova, M. M., and Lovelace, R. V. E. (2008). Oscillations of magnetohydrodynamic shock waves on the surfaces of T Tauri stars. Month. Notices RAS 388, 357-366. doi: 10.1111/j.1365-2966.2008.13394.x

Kurtz, D. W., and Marang, F. (1995). The discovery of delta Scuti pulsational variability in the pre-main-sequence Herbig $\mathrm{AE}$ star, $\mathrm{HR}$ 5999, and the discovery of rotational light variability in the remarkable He-weak BP star, HR 6000. Month. Notices R. Astron. Soc. 276, 191-198.

Kurtz, D. W., and Müller, M. (1999). Photometric confirmation of the $\delta$ Scuti pulsational variability in the pre-main-sequence Herbig Ae star HD104237. Month. Notices R. Astron. Soc. 310, 1071-1076. doi: $10.1046 / j .1365-8711.1999 .03016 . x$

Kurtz, D. W., Saio, H., Takata, M., Shibahashi, H., Murphy, S. J., and Sekii, T. (2014). Asteroseismic measurement of surface-to-core rotation in a main-sequence A star, KIC 11145123. Month. Notices RAS 444, 102-116. doi: $10.1093 / \mathrm{mnras} / \mathrm{stu} 1329$

Marconi, M., and Palla, F. (1998). The instability strip for pre-main-sequence stars. Astrophys. J. 507, L141-L144. doi: 10.1086/311704

Marconi, M., Ripepi, V., Alcalá, J. M., Covino, E., Palla, F., and Terranegra, L. (2000). Pulsation in two Herbig Ae stars: HD 35929 and V351 Ori. Astron. Astrophys. 355, L35-L38.

Morel, P., and Lebreton, Y. (2008). CESAM: a free code for stellar evolution calculations. Astrophys. Space Sci. 316, 61-73. doi: 10.1007/s10509-007-9663-9

Oudmaijer, R. D., Palacios, J., Eiroa, C., Davies, J. K., de Winter, D., Ferlet, R., et al. (2001). EXPORT: optical photometry and polarimetry of Vega-type and pre-main sequence stars. Astron. Astrophys. 379, 564-578. doi: 10.1051/0004-6361:20011331

Pápics, P. I., Briquet, M., Baglin, A., Poretti, E., Aerts, C., Degroote, P., et al. (2012). Gravito-inertial and pressure modes detected in the B3 IV CoRoT target HD 43317. Astron. Astrophys. 542:A55. doi: 10.1051/0004-6361/201218809

Pápics, P. I., Tkachenko, A., Aerts, C., Van Reeth, T., De Smedt, K., Hillen, M., et al. (2015). Asteroseismic fingerprints of rotation and mixing in the slowly pulsating B8 V star KIC 7760680. Astrophys. J. Lett. 803:L25. doi: 10.1088/2041-8205/803/2/L25

Paxton, B., Bildsten, L., Dotter, A., Herwig, F., Lesaffre, P., and Timmes, F. (2011). Modules for experiments in stellar astrophysics (MESA). Astrophys. J. Suppl. 192:3. doi: 10.1088/0067-0049/192/1/3

Pigulski, A., Kolaczkowski, Z., and Kopacki, G. (2000). Two pre-main sequence 5 Scuti stars in NGC 6823. Delta Scuti Star Newslett. 14:6.

Praderie, F., Catala, C., Czarny, J., The, P. S., and Tijn A Djie, H. R. E. (1991). Astron. Astrophys. Suppl. Ser. 89, 91-103.

Reipurth, B., Pedrosa, A., and Lago, M. T. V. T. (1996). H $\alpha$ emission in premain sequence stars. I. An atlas of line profiles. Astron. Astrophys. Suppl. 120, 229-256. doi: 10.1051/aas:1996286

Ricker, G. R., Winn, J. N., Vanderspek, R., Latham, D. W., Bakos, G. Á., Bean, J. L., et al. (2015). Transiting exoplanet survey satellite (TESS). J. Astron. Telescopes Instrum. Syst. 1:014003.

Ripepi, V., Bernabei, S., Marconi, M., Palla, F., Arellano Ferro, A., Bonanno, A., et al. (2006). A multisite photometric campaign on the pre-mainsequence $\delta$ Scuti pulsator IP Persei. Astron. Astrophys. 449, 335-343. doi: 10.1051/0004-6361:20054164

Rubinstein, A. E., Macías, E., Espaillat, C. C., Zhang, K., Calvet, N., and Robinson, C. (2018). A cavity of large grains in the disk around the group II Herbig Ae/Be Star HD 142666. Astrophys. J. 860:7. doi: 10.3847/1538-4357/aabfba

Rucinski, S. M., Matthews, J. M., Kuschnig, R., Pojmański, G., Rowe, J., Guenther, D. B., et al. (2008). Photometric variability of the T Tauri star TW Hya on time-scales of hours to years. Month. Notices RAS 391, 1913-1924. doi: 10.1111/j.1365-2966.2008.14014.x

Ruoppo, A., Marconi, M., Marques, J. P., Monteiro, M. J. P. F. G., ChristensenDalsgaard, J., Palla, F., et al. (2007). A theoretical approach for the interpretation of pulsating PMS intermediate-mass stars. Astron. Astrophys. 466, 261-268. doi: 10.1051/0004-6361:20066756

Samadi, R., Goupil, M.-J., Alecian, E., Baudin, F., Georgobiani, D., Trampedach, R., et al. (2005). Excitation of solar-like oscillations: from PMS to MS stellar models. J. Astrophys. Astron. 26:171. doi: 10.1007/BF02702325

Stahler, S. W. (1983). The birthline for low-mass stars. Astrophys. J. 274, 822-829. doi: $10.1086 / 161495$

Stahler, S. W., and Palla, F. (2005). The Formation of Stars. Weinheim: Wiley-VCH.

Stassun, K., and Wood, K. (1999). Magnetic accretion and photopolarimetric variability in classical $\mathrm{T}$ tauri stars. Astrophys. J. 510, 892-904. doi: $10.1086 / 306606$

Stauffer, J., Cody, A. M., Baglin, A., Alencar, S., Rebull, L., Hillenbrand, L. A., et al. (2014). CSI 2264: characterizing accretion-burst dominated light curves for young stars in NGC 2264. Astron. J. 147:83. doi: 10.1088/0004-6256/ $147 / 4 / 83$

Stauffer, J., Cody, A. M., Rebull, L., Hillenbrand, L. A., Turner, N. J., Carpenter, J., et al. (2016). CSI 2264: characterizing young stars in NGC 2264 with stochastically varying light curves. Astron. J. 151:60. doi: $10.3847 / 0004-6256 / 151 / 3 / 60$

Suran, M., Goupil, M., Baglin, A., Lebreton, Y., and Catala, C. (2001). Comparative seismology of pre- and main sequence stars in the instability strip. Astron. Astrophys. 372, 233-240. doi: 10.1051/0004-6361:20010485 
Thoul, A., Degroote, P., Catala, C., Aerts, C., Morel, T., Briquet, M., et al. (2013). High-precision CoRoT space photometry and fundamental parameter determination of the B2.5V star HD 48977. Astron. Astrophys. 551:A12. doi: 10.1051/0004-6361/201118756

Udry, S., and Santos, N. C. (2007). Statistical properties of exoplanets. Annu. Rev. Astron. Astrophys. 45, 397-439. doi: 10.1146/annurev.astro.45.051806. 110529

van Boekel, R., Juhász, A., Henning, T., Köhler, R., Ratzka, T., Herbst, T., et al. (2010). Variable accretion as a mechanism for brightness variations in T Tauri S. Astron. Astrophys. 517:A16. doi: 10.1051/0004-6361/200913840

Vieira, S. L. A., Corradi, W. J. B., Alencar, S. H. P., Mendes, L. T. S., Torres, C. A. O., Quast, G. R., et al. (2003). Investigation of 131 Herbig Ae/Be candidate stars. Astron. J. 126, 2971-2987. doi: 10.1086/379553

Walker, G., Matthews, J., Kuschnig, R., Johnson, R., Rucinski, S., Pazder, J., et al. (2003). The MOST asteroseismology mission: ultraprecise photometry from space. Publ. Astron. Soc. Pacific 115, 1023-1035. doi: 10.1086/377358

Waters, L. B. F. M., and Waelkens, C. (1998). Herbig Ae/Be stars. Annu. Rev. Astron. Astrophys. 36, 233-266. doi: 10.1146/annurev.astro.36.1.233

Wolk, S. J., Rice, T. S., and Aspin, C. A. (2013). Near-infrared periodic and other variable field stars in the field of the cygnus OB7 star-forming region. Astron. J. 145:113. doi: 10.1088/0004-6256/145/4/113

Zwintz, K., Fossati, L., Ryabchikova, T., Guenther, D., Aerts, C., Barnes, T. G., et al. (2014). Echography of young stars reveals their evolution. Science 345, 550-553. doi: $10.1126 /$ science. 1253645

Zwintz, K., Fossati, L., Ryabchikova, T., Kaiser, A., Gruberbauer, M., Barnes, T. G., et al. (2013). $\gamma$ doradus pulsation in two pre-main sequence stars discovered by CoRoT. Astron. Astrophys. 550:A121. doi: 10.1051/0004-6361/2012 20127

Zwintz, K., Guenther, D. B., and Weiss, W. W. (2007). Nonradial oscillations on a pre-main-sequence star. Astrophys. J. 655, 342-344. doi: 10.1086/509819

Zwintz, K., Kallinger, T., Guenther, D. B., Gruberbauer, M., Huber, D., Rowe, J., et al. (2009). MOST photometry of the enigmatic PMS pulsator HD 142666. Astron. Astrophys. 494, 1031-1040. doi: 10.1051/0004-6361:200 811116

Zwintz, K., Marconi, M., Reegen, P., and Weiss, W. W. (2005). Search for pulsating pre-main-sequence stars in NGC6383. Month. Notices RAS 357, 345-353. doi: 10.1111/j.1365-2966.2005.08655.x

Zwintz, K., Moravveji, E., Pápics, P. I., Tkachenko, A., Przybilla, N., Nieva, M.-F., et al. (2017). A comprehensive study of young B stars in NGC 2264. I. Space photometry and asteroseismology. Astron. Astrophys. 601:A101. doi: 10.1051/0004-6361/201630327

Conflict of Interest: The author declares that the research was conducted in the absence of any commercial or financial relationships that could be construed as a potential conflict of interest.

Copyright (C) 2019 Zwintz. This is an open-access article distributed under the terms of the Creative Commons Attribution License (CC BY). The use, distribution or reproduction in other forums is permitted, provided the original author(s) and the copyright owner(s) are credited and that the original publication in this journal is cited, in accordance with accepted academic practice. No use, distribution or reproduction is permitted which does not comply with these terms. 